\title{
Would routine avoidance of veno-veno bypass be possible during liver transplantation?
}

\author{
Jaemin Lee \\ Department of Anesthesiology and Pain Medicine, Seoul St. Mary's Hospital, The Catholic University of Korea School of Medicine, \\ Seoul, Korea
}

Primary failure of the liver is associated with the secondary dysfunction of virtually all other organ systems, including the cardiovascular, pulmonary, renal, and central nervous systems. Furthermore, liver transplantation is a major surgical procedure with accompanying life-threatening hemorrhage, massive transfusion, and shifts in body fluids. Such patients suffer from additional insult caused by clamping of the great vessels, including the inferior vena cava (IVC) and portal vein (PV) during the anhepatic stage. As a result, additional circulatory insufficiency to organs including the kidney, and acidosis, hypoxia, intestinal edema, and changes in gut mucosal capillary permeability may occur.

To reduce these non-physiologic insults resulting from the clamping of great vessels, veno-veno bypass (VVB) has been used during the anhepatic stage. This technique involves cannulating the inferior vena cava and the portal vein, and diverting their blood flow away from the liver and back to the right heart, usually via an axillary vein or a subclavian vein. By doing so, decompression of the portal circulation, and a reduction of congestion in the lower extremities and splanchnic circulation can be achieved.

However, the use of VVB has its own adverse effects, such as accidental decannulation, circuit clots, embolic events, prolongation of operation time, vessel injury, and coagulopathy [1]. Massive pulmonary thromboembolism resulting in fatal right heart failure, and congestion of the transplant liver has been reported [2]. Several studies have reported no clear advantage for the routine use of VVB in liver transplantation surgery $[3,4]$. Furthermore, the piggyback technique, which just tangentially clamps the recipient's suprarenal caval segment, is becoming widely used for liver transplantation surgery and allows the hemodynamic disturbances during anhepatic stage to be minimised [5]. Accordingly, the regularity of use of VVB appears to vary across institutions. Although some centers never use the technique at all, and all surgeries are performed by using a piggyback technique with no complete clamping of the IVC, many use VVB in selected cases; others use the technique on every case routinely, the exception being small pediatric patients.

At present, I think we need an in-depth analysis about the use of VVB during liver transplantation surgery. There is an interesting study associated with the use of VVB and renal function in this issue of the Korean Journal of Anesthesiology [6]. Kim et al. [6] concluded that VVB does not need to be routinely applied to maintain renal function during orthotopic liver transplantation, as long as preoperative renal function is not abnormal. They also insisted that intraoperative hemodynamic instability associated with the use of VVB could be overcome with the infusion of inotropics. However, we need to focus on the patient group included in that study. For the study, they included only the patients without preoperative renal dysfunction, which made the Child-Turcotte-Pugh classification distribution 14, 8, and 3 for A, B and C, respectively. Even though the liver transplantation surgery is often determined in the early stage of disease process these days, and the number and proportion of patients with CTP criteria A has a growing

Corresponding author: Jaemin Lee, M.D., Department of Anesthesiology and Pain Medicine, Seoul St. Mary's Hospital, The Catholic University of Korea School of Medicine, 505, Banpo-dong, Seocho-gu, Seoul 137-040, Korea. Tel: 82-2-2258-6153, Fax: 82-2-537-1951, E-mail: jmlee@ catholic.ac.kr

(c) This is an open-access article distributed under the terms of the Creative Commons Attribution Non-Commercial License (http:// creativecommons.org/licenses/by-nc/3.0/), which permits unrestricted non-commercial use, distribution, and reproduction in any medium, provided the original work is properly cited. 
trend, the majority of the patients with end-stage liver disease undergoing liver transplantation surgery belong to CTP criteria $\mathrm{C}$ at this point in time. So, the results of their study should not be extrapolated to the general population with end-stage liver disease.

Selection of the patients who do not require VVB may be difficult. Several guidelines have been proposed so far: Johnson et al. [7] proposed avoidance of VVB only when the patients hemodynamically tolerate the IVC clamping; Reddy et al. [8] reported no need of VVB in cases where the operation is done by the piggyback technique; and Veroli et al. [4] concluded that VVB is not required to maintain postoperative renal function after orthotopic liver transplantation when preoperative renal function is well maintained, which is similar to the result of Kim et al.'s study [6].

In fact, as anesthesiologists, we need to consider the following additional points regarding the routine avoidance of VVB during liver transplantation surgery. First, although the piggyback technique provides better hemodynamic outcomes, partial obstruction to flow in the IVC is inevitable, which will still lead to some congestion in the gut and lower extremities, with an increased risk of instability with the release of the clamp. Second, the increased surgical time and complications associated with the placement of the cannulas can be overcome by the placement of the return cannula in the right internal jugular vein by the anesthesia team [9]. Third, the routine use of VVB has additional benefits regarding maintenance of normothermia. Continuous warming on the bypass circuit helps maintain normothermia, which in turn helps prevent worsening of any coagulopathy [9].

As anesthesiologists, what are the benefits of routine avoi- dance of VVB during liver transplantation surgery? We shouldn't immediately dismiss using VVB until more information regarding the use of the technique has been gathered.

\section{References}

1. Shaw BW Jr, Martin DJ, Marquez JM, Kang YG, Bugbee AC Jr, Iwatsuki S, et al. Venous bypass in clinical liver transplantation. Ann Surg 1984; 200: 524-34.

2. Navalgund AA, Kang Y, Sarner JB, Jahr JS, Gieraerts R. Massive pulmonary thromboembolism during liver transplantation. Anesth Analg 1988; 67: 400-2.

3. Wall WJ, Grant DR, Duff JH, Kutt JL, Ghent CN, Bloch MS. Liver transplantation without venous bypass. Transplantation 1987; 43: 56-61.

4. Veroli P, el Hage C, Ecoffey C. Does adult liver transplantation without venovenous bypass result in renal failure? Anesth Analg 1992; 75: 489-94.

5. Miyamoto S, Polak WG, Geuken E, Peeters PM, de Jong KP, Porte $\mathrm{RJ}$, et al. Liver transplantation with preservation of the inferior vena cava: a comparison of conventional and piggyback techniques in adults. Clin Transplant 2004; 18: 686-93.

6. Kim DY, Huh IY, Cho YW, Park ES, Park SE, Nah YW, et al. Experience without using venoveno bypass in adult orthotopic liver transplantation. Korean J Anesthesiol 2011; 60: 19-24.

7. Johnson MW, Powelson JA, Auchincloss H Jr, Delmonico FL, Cosimi AB. Selective use of veno-venous bypass in orthotopic liver transplantation. Clin Transplant 1996; 10: 181-5.

8. Reddy KS, Johnston TD, Putnam LA, Isley M, Ranjan D. Piggyback technique and selective use of veno-venous bypass in adult orthotopic liver transplantation. Clin Transplant 2000; 14(4 Pt 2): 370-4.

9. Barnett R. Pro: veno-veno bypass should routinely be used during liver transplantation. J Cardiothorac Vasc Anesth 2006; 20: 742-3. 